\title{
Asymptotic Behavior of Blowup Solutions of a Parabolic Equation with the $p$-Laplacian
}

\author{
Ataru FuJII * AND Masahito OHTA**
}

\begin{abstract}
We consider the blowup problem for $u_{t}=\Delta_{p} u+|u|^{p-2} u(x \in \Omega, t>0)$ under the Dirichlet boundary condition and $p>2$. We derive sufficient conditions on blowing up of solutions. In particular, it is shown that every non-negative and non-zero solution blows up in a finite time if the domain $\Omega$ is large enough. Moreover, we show that every blowup solution behaves asymptotically like a self-similar solution near the blowup time. The Rayleigh type quotient introduced in Lemma A plays an important role throughout this paper.
\end{abstract}

\section{\$1. Introduction and Results}

In this paper we mainly consider the blowup problem for the following initial boundary value problem:

$$
\begin{cases}u_{t}=\Delta_{p} u+|u|^{q-2} u, & x \in \Omega, \quad t>0, \\ u(x, t)=0, & x \in \partial \Omega, \quad t \geq 0, \\ u(x, 0)=u_{0}(x), & x \in \Omega,\end{cases}
$$

where $p, q>2, \Delta_{p} u=\operatorname{div}\left(|\nabla u|^{p-2} \nabla u\right)$ and $\Omega$ is a bounded domain in $\mathbb{R}^{N}$ with smooth boundary $\partial \Omega$. Especially, we here study the case when $p=q$.

As for the existence and non-existence of global solutions of (1.1), the following results are well known (see[14],[9],[5],[11]):

(i) When $p>q$, (1.1) has a global solution for any $u_{0} \in W_{0}^{1, p}$.

(ii) When $p<q$, for sufficiently small initial function $u_{0} \in W_{0}^{1, p}$, (1.1) has a global solution, and if $u_{0}$ is large enough, the solution blows up in a finite time.

Communicated by T. Kawai, October 23, 1995

1991 Mathematics Subject Classification (s): 35K65, 35J20, 35J70

** Partially Supported by JSPS Research Fellowships for Young Scientists

*.**Graduate School of Mathematical Sciences, University of Tokyo, Komaba, Tokyo 153, Japan 
(iii) When $p=q$, put $\lambda_{1}=\inf \left\{\|\nabla u\|_{p}^{p} /\|u\|_{p}: u \in W_{0}^{1, p} \backslash\{0\}\right\}$. If $\lambda_{1} \geq 1$, (1.1) has a global solution for any $u_{0} \in W_{0}^{1, p}$.

Here, $W_{0}^{1, p} \equiv W_{0}^{1, p}(\Omega)$ denotes the usual Sobolev space with the norm $\|u\|_{W_{0}^{1, p}}=$ $\|\nabla u\|_{p}$, and $\|\cdot\|_{p}$ denotes the $L^{p}(\Omega)$ norm.

From the above results, we see that the case $p=q$ is critical for the existence of blowup solutions of (1.1). For the critical exponents of other equations and their role, we refer to the survey paper by Levine [8]. Here, we should note that little is known about the case when $p=q$ and $\lambda_{1}<1$. So, in what follows, we study (1.1) with the case when $p=q>2$, that is, we consider the following problem:

$$
\begin{cases}u_{t}=\Delta_{p} u+|u|^{p-2} u, & x \in \Omega, \quad t>0, \\ u(x, t)=0, & x \in \partial \Omega, \quad t \geq 0, \\ u(x, 0)=u_{0}(x), & x \in \Omega,\end{cases}
$$

Our first purpose in this paper is to derive sufficient conditions on blowing up of solutions of (P) (Theorems $B$ and $\mathrm{C}$ ). The second purpose is to study the asymptotic behavior of solutions of $(\mathrm{P})$. Here, we note that we consider not only the asymptotic behavior of blowup solutions but also that of global solutions. In both cases, we show that each solution of $(\mathrm{P})$ behaves asymptotically like a self-similar solution of $(\mathrm{P})$. First, we derive blowup rate and decay rate of solutions of $(\mathrm{P})$ for each case (Theorem $\mathrm{D})$. Next, we investigate the asymptotic profile of both blowup and global solutions of $(\mathrm{P})$ near the maximal existence time (Theorem E). These results for the case $p>2$ in (P) may be regarded as a natural extension of the linear case $p=2$ in $(\mathrm{P})$.

To be more precise, we here recall the local existence results for $(P)$. The local existence of strong solutions of $(\mathrm{P})$ is already studied by many authors (see [5], [7], [10], [12]). Here, a function $u(x, t)$ is said to be a strong solution of (P) in $[0, T]$ if (i) $u \in C\left([0, T] ; W_{0}^{1, p}(\Omega)\right)$, (ii) $u_{t}, \Delta_{p} u$ and $|u|^{p-2} u \in$ $L^{2}\left(0, T ; L^{2}(\Omega)\right)$, and (iii) $u$ satisfies (P). Assume that $p>2$, and $2(p-1) \leq N p /(N-p)$ if $p<N$. Then, for any $u_{0} \in W_{0}^{1, p}$, there exists a positive number $T$ such that $(\mathrm{P})$ has a strong solution in $[0, T]$. Moreover, let $T^{*}$ be the maximal existence time of the strong solution $u(t)$ of $(\mathrm{P})$. Then, if $T^{*}<\infty$, it follows together with (1.6) below that

$$
\lim _{t \rightarrow T^{*}}\|u(t)\|_{2}=\lim _{t \rightarrow T^{*}}\|\nabla u(t)\|_{p}=\infty .
$$

Furthermore, if we put $E(u)=\|\nabla u\|_{p}^{p}-\|u\|_{p}^{p}$, we have

$$
\partial_{t}\|u(t)\|_{2}^{2}=-2 E(u(t)) \quad \text { a.e. in } \quad\left[0, T^{*}\right) \text {, }
$$




$$
\partial_{t} E(u(t))=-p\left\|u_{t}(t)\right\|_{2}^{2} \quad \text { a.e. in } \quad\left[0, T^{*}\right) \text {, }
$$

We note that $E(\lambda u)=\lambda^{p} E(u)$ holds for any $\lambda>0$ and $u \in W_{0}^{1, p}$, which is a special feature in the critical case. Our main idea in this paper is to introduce the Rayleigh type quotient $E(u) /\|u\|_{2}$. The following lemma is important in this paper.

Lemma A. Assume that $u_{0} \in W_{0}^{1, p}$, and let $u(t)$ be a strong solution of $(P)$ in $\left[0, T^{*}\right)$. Then, we have

$$
\partial_{t} \frac{E(u(t))}{\|u(t)\|_{2}^{p}} \leq 0 \quad \text { a.e. in } \quad\left[0, T^{*}\right) \text {. }
$$

Lemma A follows immediately from (1.2) and (1.3), but it plays an essential role in the proofs of the following theorems. We should mention that a similar result to Lemma A is obtained by Berryman and Holland [1] for the fast diffusion $\left(u^{q-1}\right)_{t}=\Delta u$ with $q>2$. In [1] they study the asymptotic behavior of finite time extinction solutions of it.

First, we derive two sufficient conditions that the solution of (P) blows up in a finite time.

Theorem B. Let $p>2$ and $\lambda_{1}<1$. Assume that $u_{0} \in W_{0}^{1, p}$ satisfies $E\left(u_{0}\right)$ $<0$. Then, the strong solution of $(P)$ blows up in a finite time.

Theorem C. Let $p>2$ and $\lambda_{1}<1$. Assume that $u_{0} \in W_{0}^{1, p} \backslash\{0\}$ is non-negative in $\Omega$. Then, the strong solution of $(P)$ blows up in a finite time.

Here, we recall that $\lambda_{1}=\inf \left\{\|\nabla u\|_{p}^{p} /\left\|_{u}\right\|_{p}^{p}: u \in W_{0}^{1, p} \backslash\{0\}\right\}$, and if $\lambda_{1} \geq 1$, every strong solution of (P) exists globally in time. Theorems $B$ and $C$ supplement the known results by many authors concerning the exisence and nonexistence of global solutions of (1.1) by giving information about the case of $p=q>2$. In [2] Galaktionov showed a similar result to Theorem C for $u_{t}=\Delta u^{m}$ $+u^{m}$ with $m>1$ by using the so-called Kaplan method [6]. We should mention that this method is not applicable to our problem $(\mathrm{P})$, and our proof of Theorem $\mathrm{C}$ is quite different from that of [2].

Next, we consider the asymptotic behavior of strong solutions of $(\mathrm{P})$. We begin with deriving blowup rate and decay rate of strong solutions of $(\mathrm{P})$.

Theorem D. Assume $p>2$ and $u_{0} \in W_{0}^{1, p} \backslash\{0\}$. Let $T^{*}$ be the maximal existence time of the strong solution $u(t)$ of $(P)$. Put $\gamma_{*}=\lim _{t-T^{*}}\left[E(u(t)) /\|u(t)\|_{2}\right]$. 
(i) If $T^{*}<\infty$, we have $\gamma_{*}<0$ and

$$
\lim _{t \rightarrow T^{*}}\left[-\gamma_{*}(p-2)\left(T^{*}-t\right)\right]^{\frac{1}{p-2}}\|u(t)\|_{2}=1
$$

(ii) If $T^{*}=\infty$ and $\gamma_{*}>0$, we have

$$
\lim _{t \rightarrow \infty}\left[\gamma_{*}(p-2) t\right]^{\frac{1}{p-2}}\left\|_{u}(t)\right\|_{2}=1
$$

Remark 1.1. Put $\gamma_{1}=\inf \left\{E(u) /\left\|_{u}\right\|_{2}^{p}: u \in W_{0}^{1, p} \backslash\{0\}\right\}$. Then, we see that $\gamma_{1}>-\infty$. In fact, by the Gagliardo-Nirenberg and the Young inequalities, there exist positive constants $\alpha \in(0, p), C_{1}$ and $C_{2}$ such that

$$
\|u\|_{p}^{p} \leq C_{1}\|u\|_{2}^{p-\alpha}\|\nabla u\|_{p}^{\alpha} \leq \frac{1}{2}\|\nabla u\|_{p}^{p}+C_{2}\left\|_{u}\right\|_{2}^{p}, \quad u \in W_{0}^{1, p},
$$

from which we have

$$
\|\nabla u\|_{p}^{p} \leq 2 E(u)+2 C_{2}\left\|_{u}\right\|_{2}^{p}, \quad u \in W_{0}^{1, p}
$$

and we have $\gamma_{1} \geq-C_{2}$. So, it follows from Lemma $A$ and this fact that the limit $\gamma_{*}=\lim _{t \rightarrow T^{*}}\left[E(u(t)) /\left\|_{u}(t)\right\|_{2}\right]$ exists and $\gamma_{*} \geq \gamma_{1}$ holds for any strong solution $u(t)$ of $(\mathrm{P})$. We also note that from Theorem B, if $T^{*}=\infty$, we have $\gamma_{*} \geq 0$. Moreover, we see that $\gamma_{1}<0$ [resp. $\gamma_{1}=0, \gamma_{1}>0$ ] if and only if $\lambda_{1}<1$ [resp. $\left.\lambda_{1}=1, \lambda_{1}>1\right]$.

Remark 1.2. A function $u(x, t)=v(t) w(x)$ of variable separation type is called a self-similar solution of (P) with $u_{0}(x)=v(0) w(x)$ if $v$ and $w \in$ $W_{0}^{1, p}$ satisfy

$$
\begin{aligned}
v_{t}=-\gamma|v|^{p-2} v & \text { in } \quad \mathbb{R} \\
-\Delta_{p} w-|w|^{p-2} w=\gamma w & \text { in } \quad \mathscr{D}^{\prime}(\Omega)
\end{aligned}
$$

for some $r \in \mathbb{R}$. From Theorem $D$, we see that the blowup rate and the decay rate of general strong solutions of $(P)$ in Theorem $D$ are the same as those of the self-similar solutions of $(P)$.

Remark 1.3. In the case when $p<q$ in (1.1), the decay rate of small global solutions of (1.1) is given by H. Ishii [5]. However, it seems that in [5] there are no results for blowup rate of solutions of (1.1) when $2<p<q$. For the semilinear case $p=2<q$, see Giga and Kohn [3] and references therein. 
The following theorem states that the asymptotic profiles of solutions of (P) are given by the solutions of (1.8).

Theorem E. Assume that $p>2$ and $u_{0} \in W_{0}^{1, p} \backslash\{0\}$. Let $T^{*} \in(0, \infty]$ be the maximal existence time of the strong solution $u(t)$ of $(P)$. Then, for any sequence $\left\{t_{j}\right\}$ satisfying $t_{j} \rightarrow T^{*}$, there exist a subsequence $\left\{t_{j^{\prime}}\right\}$ of $\left\{t_{j}\right\}$ and $w \in W_{0}^{1, p}$ such that

$$
\begin{gathered}
\frac{u\left(t_{j^{\prime}}\right)}{\left\|u\left(t_{j^{\prime}}\right)\right\|_{2}} \rightarrow w \quad \text { in } W_{0}^{1, p}, \\
-\Delta_{p} w-|w|^{p-2} w=\gamma_{*} w \quad \text { in } \quad \mathscr{D}^{\prime}(\Omega), \quad\|w\|_{2}=1,
\end{gathered}
$$

where $\gamma_{*}=\lim _{t \rightarrow T^{*}}\left[E(u(t)) /\|u(t)\|_{2}\right]$

Remark 1.4. It is natural to ask in Theorem $\mathrm{E}$ whether the limit $u(t) /\|u(t)\|_{2}$ exists or not in $W_{0}^{1, p}$ as $t \rightarrow T^{*}$. At the present, we do not know the answer, even if the solution $u(t)$ of $(\mathrm{P})$ is non-negative. Of course, if nonnegative solution $w \in W_{0}^{1, p}$ of (1.10) is unique, then it follows immediately from Theorem E that $u(t) /\left\|_{u}(t)\right\|_{2} \rightarrow w$ in $W_{0}^{1, p}$ as $t \rightarrow T^{*}$ for any nonnegative and non-zero solution $u(t)$ of $(\mathrm{P})$. However, as we show in Section 3 for the case $N=1$, non-negative solution of (1.10) is not unique in general.

The plan of this paper is as follows. In Section 2 , we give the proofs of Lemma A and Theorems B, C, D and E. Lemma A will play an important role throughout this paper. Theorems B and D (ii) follow immediately from (1.2) and Lemma $A$. In order to prove Theorems $D(i)$ and $E$, we use the rescaling arguments together with Lemma A. Theorem C is proved by contradiction, using Theorem E. In Section 3, we discuss the uniqueness and non-uniqueness of non-negative solutions of (1.10) for the case $N=1$.

\section{§2. Proofs of Theorems}

In this section, we give the proofs of Lemma A and Theorems B, C, D and E. First, we give the proof of Lemma A.

Proof of Lemma A. From (1.2) and (1.3), we have

$$
\partial_{t} \frac{E(u(t))}{\|u(t)\|_{2}^{p}}=\frac{\|u(t)\|_{2}^{p} \partial_{t} E(u(t))-E(u(t)) \partial_{t}\|u(t)\|_{2}^{p}}{\|u(t)\|_{2}^{2 p}}
$$




$$
\begin{aligned}
& =\frac{-p\left\|_{u}(t)\right\|_{2}^{p}\left\|_{u_{t}}(t)\right\|_{2}^{2}+(p / 4) \partial_{t}\left\|_{u}(t)\right\|_{2}^{2}\left\|_{u}(t)\right\|_{2}^{p-2} \partial_{t}\|u(t)\|_{2}^{2}}{\|u(t)\|_{2}^{2 p}} \\
& =\frac{p\left\{\left(\partial_{t}\|u(t)\|_{2}^{2}\right)^{2}-4\|u(t)\|_{2}^{2}\left\|_{u_{t}}(t)\right\|_{2}^{2}\right\}}{4\|u(t)\|_{2}^{p+2}}
\end{aligned}
$$

a.e. in $\left[0, T^{*}\right)$. By the Cauchy-Schwarz inequality, we obtain Lemma A.

Next, we prove Theorems B and D (ii), using (1.2) and Lemma A only.

Proof of Theorem B. By Lemma A, we have

$$
\frac{E(u(t))}{\|u(t)\|_{2}^{p}} \leq \frac{E\left(u_{0}\right)}{\left\|u_{0}\right\|_{2}^{p}}, \quad t \in\left[0, T^{*}\right) .
$$

Put $c_{0}=-E\left(u_{0}\right) /\left\|u_{0}\right\|_{2}^{p}$. Then, from (1.2) and our assumption $E\left(u_{0}\right)<0$, we have $c_{0}>0$ and

$$
\partial_{t}\|u(t)\|_{2}^{2}=-2 E(u(t)) \geq 2 c_{0}\left\|_{u}(t)\right\|_{2}^{p}, \quad t \in\left[0, T^{*}\right) .
$$

Since we consider the case $p>2$, it follows from (2.1) that $T^{*}<\infty$.

Proof of Theorem $D$ (ii). From Lemma A, for any $\varepsilon>0$ there exists a $T_{\varepsilon}>0$ such that

$$
\gamma_{*} \leq \frac{E(u(t))}{\|u(t)\|_{2}^{p}} \leq \gamma_{*}+\varepsilon, \quad t \in\left[T_{\varepsilon}, \infty\right)
$$

By (1.2) and (2.2), we have

$$
-2\left(\gamma_{*}+\varepsilon\right)\left\|_{u}(t)\right\|_{2}^{p} \leq \partial_{t}\|u(t)\|_{2}^{2} \leq-2 \gamma_{*}\|u(t)\|_{2}^{p}, \quad t \in\left[T_{\varepsilon}, \infty\right)
$$

From (2.3), we get

$$
\begin{aligned}
& {\left[\left\|u\left(T_{\varepsilon}\right)\right\|_{2}^{-(p-2)}+\left(\gamma_{*}+\varepsilon\right)(p-2)\left(t-T_{\varepsilon}\right)\right]^{-\frac{2}{p-2}}} \\
& \leq\|u(t)\|_{2}^{2} \leq\left[\left\|u\left(T_{\varepsilon}\right)\right\|_{2}^{-(p-2)}+\gamma_{*}(p-2)\left(t-T_{\varepsilon}\right)\right]^{-\frac{2}{p-2}}, \quad t \in\left[T_{\varepsilon}, \infty\right),
\end{aligned}
$$

from which we have

$$
\left[\gamma_{*} /\left(\gamma_{*}+\varepsilon\right)\right]^{\frac{1}{p-2}} \leq \liminf _{t \rightarrow \infty}\left[\gamma_{*}(p-2) t\right]^{\frac{1}{p-2}}\left\|_{u}(t)\right\|_{2}
$$




$$
\leq \lim \sup _{t \rightarrow \infty}\left[\gamma_{*}(p-2) t\right]^{\frac{1}{p-2}}\|u(t)\|_{2} \leq 1
$$

Since $\varepsilon>0$ is arbitrary, we obtain (1.5).

Remark 2.1. When $T^{*}=\infty$, it follows from Theorem B that $\gamma_{*} \geq 0$. Conversely, if $\gamma_{*} \geq 0$, we have $T^{*}=\infty$. In fact, suppose that $\gamma_{*} \geq 0$. Then, it follows from the definition of $\gamma_{*}$ that $E(u(t)) \geq 0$ for any $t \in\left[0, T^{*}\right)$. From (1.2), we see that $\|u(t)\|_{2} \leq\left\|_{u_{0}}\right\|_{2}$ for any $t \in\left[0, T^{*}\right)$, from which we have $T^{*}=\infty$. In the case when $\gamma_{*}=0$, from the proof of Theorem D (ii), we see that there exists a positive constant $C_{1}$ such that $\|u(t)\|_{2} \geq C_{1}(1+t)^{-1 /(p-2)}$ for any $t \in[0, \infty)$.

Next, we prove Theorems $D(i)$ and $E$, using the rescaling arguments.

Proof of Theorem $D(i)$. First, from Remark 2.1, we see that $\gamma_{*}<0$. In order to show (1.4), we introduce the rescaled function $\bar{u}(x, \tau)$ defined by

$$
\begin{aligned}
\bar{u}(x, \tau) & =\left(T^{*}-t\right) \frac{1}{p-2} u(x, t), \quad t=T^{*}-e^{-\tau}, \\
& =\exp \left(-\frac{\tau}{p-2}\right) u\left(x, T^{*}-e^{-\tau}\right)
\end{aligned}
$$

Then, $\bar{u}(x, \tau)$ satisfies

$$
\bar{u}_{\tau}=\Delta_{p} \bar{u}+|\bar{u}|^{p-2} \bar{u}-\frac{1}{p-2} \bar{u}, \quad \tau \in\left(-\log T^{*}, \infty\right)
$$

Multiplying $(2.4)$ by $\bar{u}(x, \tau)$ and integrating over $\Omega$, we have

$$
\partial_{\tau}\|\bar{u}(\tau)\|_{2}^{2}=-2 E(\bar{u}(\tau))-\frac{2}{p-2}\|\bar{u}(\tau)\|_{2}^{2}
$$

Since we have $\lim _{\tau \rightarrow \infty}\left[E(\bar{u}(\tau)) /\|\bar{u}(\tau)\|_{2}^{p}\right]=\lim _{t \rightarrow T^{*}}\left[E(u(t)) /\|u(t)\|_{2}^{p}\right]=\gamma_{*}$, for any $\varepsilon>0$ there exists $T_{\varepsilon}>0$ such that

$$
\gamma_{*} \leq \frac{E(\bar{u}(\tau))}{\|\bar{u}(\tau)\|_{2}^{p}} \leq \gamma_{*}+\varepsilon, \quad \tau \in\left[T_{\varepsilon}, \infty\right)
$$

From (2.5), we have

$$
f_{\varepsilon}\left(\|\bar{u}(\tau)\|_{2}^{2}\right) \leq \partial_{\tau}\|\bar{u}(\tau)\|_{2}^{2} \leq f_{0}\left(\|\bar{u}(\tau)\|_{2}^{2}\right), \quad \tau \in\left[T_{\varepsilon}, \infty\right)
$$

Here we put $f_{\delta}(s)=-2\left(\gamma_{*}+\delta\right) s^{p / 2}-(2 /(p-2)) s$ for $\delta=0$ and $\varepsilon$. To conclude the proof, we have only to show that 


$$
A_{0} \leq\|\bar{u}(\tau)\|_{2}^{2} \leq A_{\varepsilon}, \quad \tau \in\left[T_{\varepsilon}, \infty\right)
$$

where $A_{\delta}=\left[-\left(\gamma_{*}+\delta\right)(p-2)\right]^{-2 /(p-2)}$ and $f_{\delta}\left(A_{\delta}\right)=0$. In fact, since $\varepsilon>0$ is arbitrary, (1.4) follows from (2.7) and the definition of $\bar{u}(x, \tau)$. We prove (2.7) by contradiction. First, suppose that there exists $\tau_{0} \in\left[T_{\varepsilon}, \infty\right)$ such that $\left\|\bar{u}\left(\tau_{0}\right)\right\|_{2}^{2}<A_{0}$. Then, from the second inequality of (2.6), we see that there exists a positive constant $C_{0}$ such that $\|\bar{u}(\tau)\|_{2}^{2} \leq C_{0} \exp \{-2 \tau /(p-2)\}$ for any $\tau \geq \tau_{0}$. Since $\|\bar{u}(\tau)\|_{2}^{2}=\exp \{-2 \tau /(p-2)\}\left\|u\left(T^{*}-e^{-\tau}\right)\right\|_{2}^{2}$, we have $\left\|u\left(T^{*}-e^{-\tau}\right)\right\|_{2}^{2}$ $\leq C_{0}$ for any $\tau \geq \tau_{0}$. However, this contradicts the fact that $\lim _{t \rightarrow T^{*}}\|u(t)\|_{2}=\infty$. Thus, we obtain the first inequality of (2.7). Next, suppose that there exists $\tau_{1}$ $\in\left[T_{\varepsilon}, \infty\right)$ such that $\left\|\bar{u}\left(\tau_{1}\right)\right\|_{2}^{2}>A_{\varepsilon}$. From the first inequality of (2.6), we see that there exists $T_{1} \in\left(\tau_{1}, \infty\right)$ such that $\lim _{\tau \rightarrow T_{1}}\|\bar{u}(\tau)\|_{2}^{2}=\infty$. However, this contradicts the fact that $\bar{u}(\tau)$ exists for all $\tau \in\left(-\log T^{*}, \infty\right)$. Thus, we obtain the second inequality of (2.7), and the proof of Theorem D (i) is completed.

Proof of Theorem E. For the solution $u(x, t)$ of $(\mathrm{P})$ in $\left[0, T^{*}\right)$, we define the rescaled function $\tilde{u}(x, \tau)$ as follows:

$$
\widetilde{u}(x, \tau)=\frac{u(x, t)}{\|u(t)\|_{2}}, \quad \tau(t)=\int_{0}^{t}\left\|_{u}(s)\right\|_{2}^{p-2} d s
$$

Then, from Theorem D and Remark 2.1, we see that $\tau\left(T^{*}\right)=\infty$ and $\tilde{u}(\tau)$ satisfies

$$
\widetilde{u}_{\tau}=\Delta_{p} \tilde{u}+|\tilde{u}|^{p-2} \tilde{u}+E(\widetilde{u}) \widetilde{u}, \quad \tau \in[0, \infty) .
$$

First, we show that for any sequence $\left\{\tau_{j}\right\}$ satisfying $\tau_{j} \rightarrow \infty$ there exist a subsequence $\left\{\tau_{j^{\prime}}\right\}$ of $\left\{\tau_{j}\right\}$ and $w \in W_{0}^{1, p}$ such that

$$
\widetilde{u}\left(\tau_{j^{\prime}}\right) \rightarrow w \quad \text { in } \quad L^{2}(\Omega)
$$

and $w$ satisfies (1.10). Since $\|\tilde{u}(\tau)\|_{2}=1$ for $\tau \in[0, \infty)$, multiplying (2.8) by $\tilde{u}_{\tau}(x, \tau)$ and integrating over $\Omega$, we have

$$
\partial_{\tau} E(\tilde{u}(\tau))=-p\left\|\tilde{u}_{\tau}(\tau)\right\|_{2}^{2}, \quad \tau \in[0, \infty) .
$$

From (2.10) and

$$
\lim _{\tau \rightarrow \infty} E(\tilde{u}(\tau))=\lim _{t \rightarrow T^{*}} \frac{E(u(t))}{\|u(t)\|_{2}^{p}}=\gamma_{*},
$$


we have $\int_{0}^{\infty}\left\|\tilde{u}_{\tau}(\tau)\right\|_{2}^{2} d \tau<\infty$. Here, following the proof of Lemma 4 of Otani [11], we set $\tilde{u}_{j}(\sigma)=\tilde{u}\left(\tau_{j}+\sigma\right)$ for $0 \leq \sigma \leq 1$. Then, we see that $\left\{\widetilde{u}_{j}\right\} \subset C([0,1]$; $\left.W_{0}^{1, p}(\Omega)\right)$, and $\tilde{u}_{j}$ satisfies

$$
\partial_{\sigma} \widetilde{u}_{j}=\Delta_{p} \widetilde{u}_{j}+\left|\widetilde{u}_{j}\right|^{p-2} \widetilde{u}_{j}+E\left(\widetilde{u}_{j}\right) \widetilde{u}_{j}, \quad \sigma \in[0,1] .
$$

It follows from $\int_{0}^{\infty}\left\|\tilde{u}_{\tau}(\tau)\right\|_{2}^{2} d \tau<\infty$ that

$$
\left\|\partial_{\sigma} \widetilde{u}_{j}\right\|_{L^{2}\left(0,1 ; L^{2}(\Omega)\right)} \rightarrow 0 .
$$

Moreover, since $\left\|\tilde{u}_{j}(\sigma)\right\|_{2}=1$ for $\sigma \in[0,1]$, it follows from (1.6) and (2.10) that

$$
\sup _{j}\left\|\tilde{u}_{j}\right\|_{L^{\infty}\left(0,1 ; W_{0}^{1, p}(\Omega)\right)}<\infty .
$$

By $(2.11)-(2.14)$, the monotonicity of $-\Delta_{p}$ and the standard compactness argument, we see that there exist a subsequence $\left\{\widetilde{u}_{j^{\prime}}\right\}$ of $\left\{\widetilde{u}_{j}\right\}$ and $\widetilde{w} \in L^{\infty}(0,1$; $\left.W_{0}^{1, p}(\Omega)\right)$ such that

$$
\tilde{u}_{j^{\prime}} \rightarrow \widetilde{w} \quad \text { in } \quad C\left([0,1] ; L^{2}(\Omega)\right)
$$

and $\widetilde{w}(\sigma)$ satisfies $(1.10)$ for each $\sigma \in[0,1]$ (see the proofs of Theorem 1 of [14] and Lemma 4 of [11]). Putting $w=\widetilde{w}(0)$, we see that there exists a subsequence $\left\{\tau_{j^{\prime}}\right\}$ of $\left\{\tau_{j}\right\}$ satisfying (2.9) and $w$ satisfies (1.10). Finally, we show that there exists a subsequence $\left\{\tau_{j^{\prime \prime}}\right\}$ of $\left\{\tau_{j^{\prime}}\right\}$ such that

$$
\tilde{u}\left(\tau_{j^{\prime \prime}}\right) \rightarrow w \quad \text { in } \quad W_{0}^{1, p}(\Omega) .
$$

In fact, since $\left\{\tilde{u}\left(\tau_{j^{\prime}}\right)\right\}$ is bounded in $W_{0}^{1, p}$, it follows from (2.9) that there exists a subsequence $\left\{\tau_{j^{\prime \prime}}\right\}$ of $\left\{\tau_{j^{\prime}}\right\}$ such that

$$
\widetilde{u}\left(\tau_{j^{\prime \prime}}\right) \rightarrow w \quad \text { weakly in } W_{0}^{1, p}(\Omega) \text { and strongly in } L^{p}(\Omega)
$$

Since $w$ satisfies (1.10), it follows from (2.11) that $E\left(\tilde{u}\left(\tau_{j^{\prime \prime}}\right)\right) \rightarrow \gamma_{*}=E(w)$. Moreover, it follows from (2.16) that $\left\|\widetilde{u}\left(\tau_{j^{\prime \prime}}\right)\right\|_{p}^{p} \rightarrow\|w\|_{p}^{p}$. Thus, we have

$$
\left\|\nabla \widetilde{u}\left(\tau_{j^{\prime \prime}}\right)\right\|_{p}^{p} \rightarrow\|\nabla w\|_{p}^{p}
$$

Since $W_{0}^{1, p}$ is a uniformly convex Banach space, (2.15) follows from (2.16) and (2.17). This completes the proof of Theorem E. 
Finally, we prove Theorem C. To prove it, we need to prepare one lemma.

Lemma 2.2. Let $p>2, \lambda_{1}>1$ and $\gamma \geq 0$. Suppose that $w \in W_{0}^{1, p}$ is nonnegative in $\Omega$, and satisfies $-\Delta_{p} w-|w|^{p-2} w=\gamma w$ in $\mathscr{D}^{\prime}(\Omega)$. Then, we have $w \equiv 0$ in $\Omega$.

Proof of Lemma 2.2. Suppose that $w \neq 0$ in $\Omega$. Then, by the standard argument (see, e.g., $\left[13\right.$, p.418]), we see that $w \in C^{1+\alpha}(\bar{\Omega})$ for some $\alpha \in(0,1)$ and $w$ is positive in $\Omega$. Let $\varphi$ be a positive solution of $-\Delta_{p} \varphi=\lambda_{1}|\varphi|^{p-2} \varphi$ in $\mathscr{D}^{\prime}(\Omega)$. Since $w$ satisfies $-\Delta_{p} w \geq|w|^{p-2} w$ in $\mathscr{D}^{\prime}(\Omega)$, in the same way as in the proof of Theorem II of [4], we get $\varphi \equiv 0$ in $\Omega$. This is a contradiction. Hence, we have $w \equiv 0$ in $\Omega$.

Proof of Theorem $C$. We prove by contradiction. Let $u(t)$ be a global solution of (P) such that $u_{0} \in W_{0}^{1, p} \backslash\{0\}$ is non-negative in $\Omega$. Then, by the maximum principle as in [14], $u(t)$ is non-negative in $\Omega$ for $t \in[0, \infty)$. From Theorem B, we have $\gamma_{*}=\lim _{t \rightarrow \infty}\left[E(u(t)) /\|u(t)\|_{2}^{p}\right] \geq 0$. Moreover, from Theorem E, there exist a sequence $\left\{t_{j}\right\}$ satisfying $t_{j} \rightarrow \infty$ and $w \in W_{0}^{1, p}$ such that

$$
\begin{gathered}
\frac{u\left(t_{j}\right)}{\left\|u\left(t_{j}\right)\right\|_{2}} \rightarrow w \quad \text { in } \quad W_{0}^{1, p}, \\
-\Delta_{p} w-|w|^{p-2} w=\gamma_{*} w \quad \text { in } \quad \mathscr{D}^{\prime}(\Omega) .
\end{gathered}
$$

Since $u(t)$ is non-negative in $\Omega$ for $t \in[0, \infty)$, from (2.18), we see that $w$ is also non-negative in $\Omega$. Thus, it follows from Lemma 2.2 that $w \equiv 0$ in $\Omega$. However, this contradicts $\|w\|_{2}=1$. Hence, we obtain Theorem $\mathrm{C}$.

\section{§3. Eigenvalue Problem (1.10) for $N=\mathbb{1}$}

In this section, we consider the eigenvalue problem (1.10) for the case $N=1$. Especially, we are interested in the set of all non-negative solutions of (1.10) with $\gamma_{*}<0$, which is related to the asymptotic profiles of non-negative blowup solutions of $(\mathrm{P})$.

First, we consider the following boundary value problem:

$$
\left\{\begin{array}{l}
-\left(\left|u^{\prime}\right|^{p-2} u^{\prime}(x)\right)^{\prime}-|u|^{p-2} u(x)=-u(x), \quad x \in \Omega \\
u \in W_{0}^{1, p}(\Omega), \quad u(x) \geq 0, \not \equiv 0, \quad x \in \Omega .
\end{array}\right.
$$


Here, the symbol ' denotes the differentiation with respect to $x$. Let $S_{l}$ be the set of all solutions of (3.1) for $\Omega=(-l, l)$. Then, the structure of $S_{l}$ is determined as follows.

Proposition 3.1. Let $l_{p}$ be the positive number such that

$$
\lambda_{1}\left(-l_{p}, l_{p}\right)=\inf \left\{\frac{\left\|u^{\prime}\right\|_{p}^{p}}{\|u\|_{p}^{p}}: u \in W_{0}^{1, p}\left(-l_{p}, l_{p}\right), u \neq 0\right\}=1
$$

and $m_{p}=p l_{p} /(p-2)$.

(1) If $l \leq l_{p}$, then $S_{l}$ is empty.

(2) If $l_{p}<l \leq m_{p}$, then there exists a unique positive solution $\Phi_{l}$ of (3.1) and $S_{l}=\left\{\Phi_{l}\right\}$.

(3) If $l>m_{p}$, then $S_{l}=\cup_{k=1}^{\left[l / m_{p}\right]} S_{l}^{k}$, where $\left[l / m_{p}\right]$ denotes the largest integer not exceeding $l / m_{p}$, and $S_{l}^{k}=\left\{\sum_{j=1}^{k} \Phi_{m_{p}}\left(\cdot-y_{j}\right):-l \leq y_{1}-m_{p}, y_{j}+2 m_{p} \leq y_{j+1}, j=\right.$ $\left.1, \ldots, k-1, y_{k}+m_{p} \leq l\right\}$.

As a corollary to Proposition 3.1, we have the main result in this section.

Theorem 3.2. Let $\gamma<0$ and $\sum(\gamma)$ be the set of all solutions of

$$
\left\{\begin{array}{l}
-\left(\left|u^{\prime}\right|^{p-2} u^{\prime}(x)\right)^{\prime}-|u|^{p-2} u(x)=\gamma u(x), \quad x \in(-l, l), \\
u \in W_{0}^{1, p}(-l, l), \quad\|u\|_{2}=1, \quad u(x) \geq 0, \quad x \in(-l, l) .
\end{array}\right.
$$

(1) When $l \leq l_{p}, \sum(\gamma)$ is empty for any $\gamma<0$.

(2) When $l_{p}<l \leq m_{p}$, let $\gamma_{1}=E\left(\Phi_{l}\right) /\left\|\Phi_{l}\right\|_{2}^{p}$. Then $\gamma_{1}<0$ and $\sum\left(\gamma_{1}\right)=\left\{\widetilde{\Phi}_{l}\right\}$, where $\widetilde{\Phi}_{l}=\Phi_{l} /\left\|\Phi_{l}\right\|_{2}$, and $\sum(\gamma)$ is empty if $\gamma \neq \gamma_{1}$.

(3) When $l>m_{p}$, for $k=1,2, \ldots,\left[l / m_{p}\right]$, let $\gamma_{k}=k^{1-p / 2} E\left(\Phi_{m_{p}}\right) /\left\|\Phi_{m_{p}}\right\|_{2}$.

Then $\gamma_{1}<\gamma_{2}<\cdots<\gamma_{\left[l / m_{p}\right]}<0$ and $\sum\left(\gamma_{k}\right)=\left\{\sum_{j=1}^{k} \widetilde{\Phi}_{m_{p}}\left(\cdot-y_{j}\right):-l \leq y_{1}-m_{p}\right.$, $\left.y_{j}+2 m_{p} \leq y_{j+1}, j=1, \ldots, k-1, y_{k}+m_{p} \leq l\right\}$, and $\sum(\gamma)$ is empty if $\gamma^{\notin}\left\{\gamma_{1}, \gamma_{2}, \ldots, \gamma_{\left[l / m_{p}\right]}\right\}$.

Theorem 3.2 follows immediately from Proposition 3.1. We note that $\gamma_{1}$ defined in Remark 1.1 coincides with that in Theorem 3.2 in this case. In order to prove Proposition 3.1, we consider the following initial value problem:

$$
\begin{cases}\left(\left|u^{\prime}\right|^{p-2} u^{\prime}(x)\right)^{\prime}= & u(x)-|u|^{p-2} u(x), \quad x>0 \\ u(0)=\alpha>0, & u^{\prime}(0)=0 .\end{cases}
$$


Lemma 3.3. Let $\alpha_{p}=(p / 2)^{1 /(p-2)}$ and $F(s)=(p /(p-1))\left(|s|^{2} / 2-|s|^{p / p}\right)$, and let $x_{\alpha}=\infty$ if $\alpha<\alpha_{p}$, and $x_{\alpha}=\int_{0}^{\alpha}[F(s)-F(\alpha)]^{-1 / p} d s$ if $\alpha \geq \alpha_{p}$. For $\alpha>0$, there exists a unique solution $\varphi_{\alpha}$ of $(3.2)$ in $\left(0, x_{\alpha}\right)$, and $\varphi_{\alpha}$ is positive in $\left(0, x_{\alpha}\right)$. Moreover, when $\alpha \geq \alpha_{p}, x_{\alpha}<\infty$ and $\varphi_{\alpha}$ satisfies $\varphi_{\alpha}\left(x_{\alpha}\right)=0, \varphi_{\alpha}^{\prime}\left(x_{\alpha}\right)<0$ if $\alpha>\alpha_{p}$, and $\varphi_{\alpha}^{\prime}\left(x_{\alpha}\right)=0$ if $\alpha=\alpha_{p}$.

Proof of Lemma 3.3. Let $u(x)$ be a solution of (3.2). Then, we have

$$
\left|u^{\prime}\right|^{p-2} u^{\prime}(x)=\int_{0}^{x}\left[u(y)-|u|^{p-2} u(y)\right] d y, \quad x \geq 0 .
$$

When $\alpha=1$, it follows from (3.3) that $u(x)=1$ for $x \geq 0$. When $\alpha \neq 1$, from (3.3) we see that there exists $x_{0}>0$ such that $(\alpha-1) u^{\prime}(x)<0$ for $0<x<x_{0}$. Thus, $u(x)$ is twice differentiable in $\left(0, x_{0}\right)$. Multiplying the equation of (3.2) by $u^{\prime}$ and integrating over $(0, x)$ yields

$$
\left|u^{\prime}(x)\right|^{p}=F(u(x))-F(\alpha), \quad x \geq 0
$$

From (3.3) and (3.4), we see that there exists a unique solution $\varphi_{\alpha}$ of (3.2) in $\left(0, x_{\alpha}\right)$, and $\varphi_{\alpha}$ is positive in $\left(0, x_{\alpha}\right)$. In particular, when $\alpha \geq \alpha_{p}, u=\varphi_{\alpha}(x)$ is given as the inverse function of $x=\int_{u}^{\alpha}[F(s)-F(\alpha)]^{-1 / p} d s$. So, we see that $x_{\alpha}<\infty$ and $\varphi_{\alpha}$ satisfies $\varphi_{\alpha}\left(x_{\alpha}\right)=0, \varphi_{\alpha}^{\prime}\left(x_{\alpha}\right)<0$ if $\alpha>\alpha_{p}$, and $\varphi^{\prime}{ }_{\alpha}\left(x_{\alpha}\right)=0$ if $\alpha=\alpha_{p}$.

Remark 3.4. By an elementary computation, we see that $x_{\alpha}$ is strictly decreasing with respect to $\alpha \geq \alpha$. It is known that $l_{p}=(p-1)^{1 / p} B(1 / p, 1-1 / p) / p$ $=\left[\pi(p-1)^{1 / p}\right] /[p \sin (\pi / p)]$, where $B(\cdot, \cdot)$ is the beta function. Another elementary calculation yields $\lim _{\alpha \rightarrow \infty} x_{\alpha}=l_{p}$ and $x_{\alpha_{p}}=m_{p}$.

Proposition 3.1 follows from Lemma 3.3 and Remark 3.4. In particular, $\Phi_{l}$ is given by

$$
\Phi_{l}(x)= \begin{cases}\varphi_{\alpha(l)}(x), & \text { for } 0 \leq x \leq l, \\ \varphi_{\alpha(l)}(-x), & \text { for }-l \leq x<0\end{cases}
$$

where $\alpha(l) \in\left[\alpha_{p}, \infty\right)$ is the unique number such that $l=x_{\alpha(l)}$. 


\section{Acknowledgement}

The authors would like to express their deep gratitude to Professor Yoshio Tsutsumi for his kind advice.

\section{References}

[1] Berryman, J. G. and Holland, C. J., Stability of the separable solution for fast diffusion, Arch. Rat. Mech. Anal., 74 (1980), 379-388.

[2] Galaktionov, V. A., Boundary-value problem for the nonlinear parabolic equation $u_{t}=\Delta u^{\sigma+1}$ $+u^{\beta}$, Differential Equations, 17 (1981), 551-555.

[3] Giga, Y. and Kohn, R. V., Characterizing blowup using similarity variables, Indiana Univ. Math. J., 36 (1987), 1-40.

[4] Idogawa, T. and Ôtani, M., The first eigenvalues of some abstract elliptic operators, Funk. Ekva., 38 (1995), 1-9.

[5] Ishii, H., Asymptotic stability and blowing up of solutions of some nonlinear equations, J. Differential Equations, 26 (1977), 291-319.

[6] Kaplan, S., On the growth of solutions of quasilinear parabolic equations, Comm, Pure Appl. Math., 16(1963), 305-330.

[7] Koi, Y. and Watanabe, J., On nonlinear evolution equations with a difference term of subdifferentials, Proc. Japan Acad., 52 (1976), 413-416.

[8] Levine, H. A., The role of critical exponents in blowup theorems, SIAM Review, 32 (1990), 262-288.

[9] Levine, H. A. and Payne, L. E., Nonexistence of global weak solutions of classes of nonlinear wave and parabolic equations, J. Math, Anal. Appl., 55 (1976), 329-334.

[10] OOtani, M., On existence of strong solutions for $d u(t) / d t+\partial \psi^{1}(u(t))-\partial \psi^{2}(u(t)) \ni f(t), J$. Fac. Sci. Univ. Tokyo Sec. IA, 24 (1977), 575-605.

[11] - Existence and asymptotic stability of strong solutions of nonlinear evolution equations with a difference term of subdifferentials, Qualitative Theory of Differential Equations, North-Holland, Colloq. Math. Soc. Janos Bolyai, 30 (1981), 795-809.

[12] - Non-monotone perturbations for nonlinear parabolic equations associated with subdifferential operators: Cauchy problem, J. Differential Equations, 46 (1982), 268-299.

[13] Sakaguchi, S., Concavity properties of solutions to some degenerate quasilinear elliptic Dirichlet problems, Ann. Scuola Norm. Sup. Pisa, Cl. Sci., Serir IV, 14(1987), 403-421.

[14] Tsutsumi, M., Existence and nonexistence of global solutions for nonlinear parabolic equations, Publ. RIMS, Kyoto Univ., 8(1972), 211-229. 
